\title{
Result and pedigree analysis of spontaneously abortion villus chromosome detecting by FISH
}

N. An, L.-L. Li, X.-Y. Zhang, W.-T. Sun, M.-H. Liu and R.-Z. Liu

Center for Reproductive Medicine of the First Bethune Hospital of Jilin University, Changchun, Jilin, China

Corresponding author: R.-Z. Liu

E-mail: Irz410@126.com

Genet. Mol. Res. 14 (4): 16662-16666 (2015)

Received May 12, 2015

Accepted June 18, 2015

Published December 11, 2015

DOI http://dx.doi.org/10.4238/2015.December.11.13

\begin{abstract}
The aim of this study was to evaluate the relationship between fetal karyotype and parental chromosomal abnormalities, and to provide a basis for clinical diagnosis and therapy in Northeast China. A total of 144 spontaneously aborted fetuses were analyzed by FISH to test for chromosome number and to recall couples for peripheral blood karyotype analysis. The rate of abnormal chorionic villus chromosomes was $35.42 \%$. Villus chromosome abnormality rate of the first spontaneous abortion and repeated abortions were 40.54 and $33.64 \%$, respectively $(P<0.05)$. The rate of chromosome abnormality in women with advanced maternal age and women younger than 35 years old were 46.43 and $32.76 \%$, respectively $(P<0.05)$. In a recall of 112 couples for peripheral blood karyotype analysis, just 3 cases of 7 patients with peripheral blood chromosome abnormality showed abnormal FISH
\end{abstract}


results in their abortion villi. Fetal chromosome number abnormality is a major cause of early abortion, and parental chromosomal abnormality is not the main factor in abnormal fetal karyotype. A complete evaluation and special treatment should be provided to couples with a history of recurrent miscarriage.

Key words: Chromosomal abnormality; Spontaneous abortion; Amniocentesis; Genetic counseling

\section{INTRODUCTION}

Almost $15-20 \%$ of all pregnancies end up in a spontaneous abortion (SA), in which the incidence of chromosomal abnormalities is as high as 70\% (Usha et al., 2011). Recurrent miscarriage $(\mathrm{RM})$ is defined as the occurrence of two or more consecutive pregnancy losses. RM occurs in 1 to $5 \%$ of all couples trying to conceive (Hogge et al., 2003). About 50 to $60 \%$ of all miscarriages are associated with cytogenetic abnormalities. Therefore, our study aimed to determine the frequency and distribution of fetal chromosomal abnormalities from miscarriages in couples with SA or RM and to see whether or not there was any difference in the frequency and distribution of chromosome abnormalities between the first pregnancy loss and two or more subsequent losses.

\section{MATERIAL AND METHODS}

This study involved a retrospective evaluation of fluorescence in situ hybridization (FISH) results of chorionic villus samples in 144 SA cases at the First Bethune Hospital of Jilin University between September 2012 and April 2014. The median age of the mothers was 31.0 (range 23-41 years). The gestational age (fetal demise at 6-12 weeks gestation) at the time of pregnancy loss was estimated by reviewing ultrasound. Written informed consent was obtained from all study subjects prior to enrollment in the study.

FISH was performed using commercially available whole-chromosome painting probes for chromosomes 13, 16, 18, 21, 22, $\mathrm{X}$ and $\mathrm{Y}$. Chromosome denaturation, hybridization, and signal detection were done according to published protocols (Licher et al., 1988). Peripheral blood karyotyping was performed in parents. Chromosomal abnormalities were reported according to the current international standard nomenclature. In all tables, data are reported as number of cases and percentage. Comparisons between groups were conducted using the chi-square test, and $\mathrm{P}<0.05$ was considered to be statistically significant in all tests.

\section{RESULTS}

Among the 144 cases of SA, villus chromosome analysis revealed 51 cases of abnormal signals, indicating a $35.42 \%$ rate of chromosomal abnormality in the chorion villus samples. Among these 51 cases, there were 12 cases of trisomy 16 and monosomy X, 7 cases of trisomy 22, 5 cases of trisomy 13 , and 4 cases of triploid and tetraploid (details are given in Table 1). 
Table 1. Total chromosomal abnormalities in chorionic villus sample among 144 cases of spontaneous abortion.

\begin{tabular}{lccc}
\hline Abnormality & Number $(\mathrm{N})$ & Proportion in abnormal cases $(\%)$ & Proportion in total cases $(\%)$ \\
\hline Trisomy 16 & 12 & 23.53 & 8.33 \\
Monosomy X & 12 & 25.49 & 9.03 \\
Trisomy 22 & 7 & 13.73 & 4.86 \\
Trisomy 13 & 5 & 9.80 & 3.47 \\
triploid & 4 & 7.84 & 2.78 \\
tetraploid & 4 & 7.84 & 2.78 \\
Trisomy 18 & 2 & 3.92 & 1.39 \\
Trisomy 21 & 2 & 3.92 & 1.39 \\
Monosomy 21 & 1 & 1.96 & 0.69 \\
Trisomy 16/22 & 1 & 1.96 & 0.69 \\
Trisomy 21/22 & 1 & 1.96 & 0.69 \\
\hline
\end{tabular}

In Table 2, data are divided into SA and RM groups. There were 37 cases in the RM group and 10 cases were abnormal, showing a rate of chromosomal abnormality of $40.54 \%$. In the SA group, there were 41 cases of numerical chromosomal abnormality in a total of 107 cases, showing a rate of chromosomal abnormality of $33.64 \%$. The SA and RM groups showed no statistically significant difference $(P<0.05)$.

Table 2. Outcome of FISH in chorionic villus chromosomes according to the frequency of miscarriages.

\begin{tabular}{lccccc}
\hline Group & Number $(\mathrm{N})$ & Abnormal $(\mathrm{N})$ & Rate $(\%)$ & $\chi^{2}$ & $\mathrm{P}$ \\
\hline RM & 37 & 15 & 40.54 & 0.57 & 0.45 \\
SA & 107 & 36 & 33.64 & & \\
\hline $\mathrm{P}<0.05$ & & & &
\end{tabular}

The outcome of FISH in the SA or RM group with aneuploidy was analyzed according to maternal age (Table 3 ). There were 28 cases in the advanced maternal age ( $\geq 35$ years old) group, of which 18 cases had a numerical abnormality (46.43\%). The younger maternal age group (<35 years old) had 166 cases, of which 38 cases had a numerical abnormality (32.76\%). The two age groups showed no statistical difference $(P<0.05)$ (Table 3$)$.

Table 3. Outcome of FISH in chorionic villus chromosomes according to maternal age.

\begin{tabular}{lccccc}
\hline Group & Number $(\mathrm{N})$ & Abnormal $(\mathrm{N})$ & Rate $(\%)$ & $\chi^{2}$ & 1.84 \\
\hline$\geq 35$ & 28 & 13 & 46.43 & 32.76 & \\
\hline 35 & 116 & 38 & 32.18 \\
\hline
\end{tabular}

$\mathrm{P}<0.05$.

Among the 144 cases of abortuses, 70 were male, including 22 with numerical abnormality (31.43\%). The frequency of female abortuses with chromosomal abnormality was 29/74 (39.19\%). There was no statistical difference between the two groups (Table 4).

Table 4. Outcome of FISH in chorionic villus chromosomes according to gender.

\begin{tabular}{lccccc}
\hline Group & Number $(\mathrm{N})$ & Abnormal $(\mathrm{N})$ & Rate $(\%)$ & $\chi^{2}$ & $\mathrm{P}$ \\
\hline Male & 70 & 22 & 31.43 & 0.95 & 0.33 \\
Female & 74 & 29 & 39.19 & & \\
\hline P & 74.05 & & & \\
\hline
\end{tabular}

$\mathrm{P}<0.05$. 
To determine whether fetal chromosomal abnormality was inherited or de novo, all parents were informed about the importance of testing their genetic material. We only collected 112 parents' peripheral blood lymphocytes for karyotype analysis, and 7 karyotypes were found to be abnormal (Table 5).

Table 5. Lymphocyte karyotype analysis of 7 couples.

\begin{tabular}{llll}
\hline Number & FISH & Cytogenetic diagnosis (female) & Cytogenetic diagnosis (male) \\
\hline 1 & Normal & $46, \mathrm{XX}$,inv $(9)(\mathrm{p} 11 \mathrm{q} 13)$ & $46, \mathrm{XY}$ \\
2 & Normal & $46, \mathrm{XX}$ & $46, \mathrm{XY}, \mathrm{inv}(9)(\mathrm{p} 11 \mathrm{q} 13)$ \\
3 & Normal & $46, \mathrm{XX}, 15 \mathrm{p}+$ & $46, \mathrm{XY}$ \\
4 & Normal & $46, \mathrm{XX}, \mathrm{t}(4 ; 11)(\mathrm{q} 21 ; \mathrm{q} 23)$ & $46, \mathrm{XY}$ \\
5 & Trisomy 16 & $46, \mathrm{XX}, 1 \mathrm{qh}+$ & $46, \mathrm{XY}$ \\
6 & Trisomy 16/22 & $46, \mathrm{XX}$ & $46, \mathrm{XY}, 16 \mathrm{qh}+$ \\
7 & Trisomy 21/22 & $46, \mathrm{XX}$ & $46, \mathrm{XY}, \mathrm{Yqh}+$ \\
\hline
\end{tabular}

\section{DISCUSSION}

Recently, there has been a gradual increase in the incidence of SA in China. Approximately $31 \%$ of SA occur in embryo implantation, and among these, $80 \%$ are early abortion (Pisani et al., 2002). About $15 \%$ of all clinically recognized pregnancies are spontaneously aborted and $60-70 \%$ of these are attributable to detectable chromosome abnormalities (Philipp et al., 2003). Trisomies have been reported in all human chromosomes; the most common ones are in chromosomes $16,21,18,13,15,22$, and $X$, accounting for $90 \%$ of trisomies (Sullivan et al., 2004). Trisomy 16 accounts for approximately $1 / 3$, and is associated with high mortality. We detected 30 cases of chromosome trisomy and $40 \%$ were trisomy 16 . Polyploidy mainly originates from fertilization by polyspermy or postzygotic division error (Simpson, 2007).

In our population of RA patients, abortus aneuploidy occurred (40.54\%) more than in sporadic miscarriages (33.64\%), but there was no statistically significant difference between the two groups, illustrating that the incidence of embryo chromosomal abnormalities showed no obvious correlation with the frequency of SA. Therefore, clinically, we recommend testing chorionic villus chromosomes in patients with SA in time, not only for normal pregnancy further strive for the time, but also to reduce patient stress (especially in recurrent SA). Furthermore, the frequency of villus chromosomal abnormality was a little higher in advanced maternal age than in maternal age younger than 35 , but there was no statistically significant difference between the two groups, showing that advanced maternal age may not be the main cause of embryonic chromosomal aneuploidy.

In addition, the frequency of male and female abortuses in spontaneous abortion was, respectively, 48.6 and $51.4 \%$, and embryonic chromosomal aneuploidy was, respectively, 43.2 and $56.9 \%$. The two groups showed no statistically significant difference. These results differed from those of Xiong et al. (2009), who found that in both SA and embryonic chromosomal aneuploidy, female embryos were significantly more frequent than male embryos. We believe that this discrepancy can be resolved with further studies with more patient samples.

In addition, we collected 112 parents' peripheral blood lymphocytes for karyotype analysis, and 7 karyotypes were abnormal, among which only 3 chorionic villus samples showed chromosomal abnormalities, indicating that vertical transmission was not the main cause of embryonic chromosomal aneuploidy. Nevertheless, errors in meiosis or mitosis in embryonic germ cells or generative cells leading to chromosome aberration are the main cause. However, 
embryonic chromosomal aneuploidy may occur repeatedly in parents with abnormal chromosomes, ultimately leading to abortion. Therefore, these patients can be considered for preimplantation genetic diagnosis/screening to have a healthy baby.

In conclusion, embryonic chromosomal aneuploidy is the main cause of early spontaneous abortion, and a detailed embryoscopic examination of the dead embryo is likely to be useful in couples who have experienced recurrent abortion. In such cases, chromosome analysis is generally recommended (Wolf and Horger, 1995).

\section{REFERENCES}

Hogge WA, Byrnes AL, Lanasa MC, Surti U, et al. (2003). The clinical use of karyotyping spontaneous abortions. Am. J. Obstet. Gynecol. 189: 397-400.

Licher P, Cremer T, Borden J, Manuelidis L, et al. (1988). Delineation of individual human chromosomes in metaphase and interphase cells by in situ suppression hybridization using recombinant DNA libraries. Hum. Genet. 80: 224-234.

Philipp T, Philipp K, Reiner A, Beer F, et al. (2003). Embryoscopic and cytogenetic analysis of 233 missed abortions: factors involved in the pathogenesis of developmental defects of early failed pregnancies. Hum. Reprod. 18: 1727-1732.

Pisani P, Bray F and Parkin DM (2002). Estimates of the world-wide prevalence of cancer for 25 sites in the adult population. Int. J. Cancer 97: 72-81.

Simpson JL (2007). Causes of fetal wastage. Clin. Obstet. Gynecol. 50: 10-30.

Sullivan AE, Silver RM, LaCoursiere DY, Porter TF, et al. (2004). Recurrent fetal aneuploidy and recurrent miscarriage. Obstet. Gynccol. 104: 784-788.

Usha R, Dutta P, Rajitha, Dalal AB, et al. (2011). Cytogenetic abnormalities in 1162 couples with recurrent miscarriages in Southern region of India: report and review. J. Assist. Reprod. Genet. 28:145-149.

Xiong L, Liu J, Deng K, Liu SP, et al. (2009). Chorionic villus cell culture and karyotype analysis in 110 cases of early spontaneous abortion. J. South Med. Univ. 29: 64-67.

Wolf GC and Horger EO 3rd (1995). Indication for examination of spontaneous abortion specimens: a reassessment. Am. J. Obstet. Gynecol. 5: 1364-1367. 